\title{
ECT, rTMS, and deepTMS in pharmacoresistant drug-free patients with unipolar depression: a comparative review
}

This article was published in the following Dove Press journal:

Neuropsychiatric Disease and Treatment

13 January 2012

Number of times this article has been viewed

\section{Amedeo Minichino' \\ Francesco Saverio Bersani' \\ Enrico Capra' \\ Rossella Pannese' \\ Celeste Bonanno ${ }^{2}$ \\ Massimo Salviati' \\ Roberto Delle Chiaie' \\ Massimo Biondi' \\ 'Department of Neurology and Psychiatry, Sapienza University of Rome, Rome, ${ }^{2}$ Aldo Moro University of Bari, Bari, Italy}

Video abstract

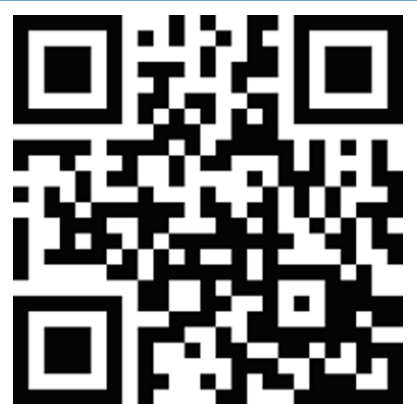

Point your SmartPhone at the code above. If you have a QR code reader the video abstract will appear. Or use: http://dvpr.es//54BQh
Correspondence: Francesco Saverio Bersani Via di Torre Argentina 2I, Rome, Italy

$\mathrm{Tel}+393405165865$

Fax $+3906499 / 4728$

Email bersani.fs@tiscali.it
Background: Biological treatments are considered as additional options for the treatment of resistant unipolar depression. Controversial data exist about the efficacy and tolerability of three of the most used somatic treatments: electroconvulsive therapy (ECT), transcranial magnetic stimulation (rTMS), and deep transcranial magnetic stimulation (deepTMS). The aim of this review is to investigate and compare the efficacy and tolerability of these three techniques in drug-free patients with pharmacoresistant unipolar depression.

Methods: Three independent reviewers extracted data and assessed the quality of methodological reporting of selected studies. The first outcome was the clinical response to the three different techniques defined as a percentage improvement of Hamilton Depression Rating Scale (HDRS). The second outcome was the evaluation of their neuropsychological effects. The third outcome was the evaluation of the number of remitted patients; remission was defined as an absolute HDRS-24 score of $\leq 11$ or as an absolute HDRS-17 score of $\leq 8$. Tolerability was the fourth outcome; it was evaluated by examining the number of dropped-out patients.

Results: The comparative evaluation of HDRS percentage variations shows ECT as the most effective method after 4 weeks of therapy; on the other hand, a better efficacy is obtainable by deepTMS after 2 weeks of therapy. DeepTMS is the technique that gives the best improvement of cognitive performances. The percentage of remitted patients obtained with ECT treatment is the same obtained in the deepTMS group. Both techniques have a remitted patients percentage two times larger than the rTMS. DeepTMS shows a tolerability, measured by the number of dropped-out patients, worse than ECT.

Conclusion: Our investigation confirms the great therapeutic power of ECT. DeepTMS seems to be the only therapy that provides a substantial improvement of both depressive symptoms and cognitive performances; nevertheless it is characterized by a poor tolerability. rTMS seems to provide a better tolerability for patients, but its therapeutic efficacy is lower. Considering the small therapeutic efficacy of deepTMS in the last 2 weeks of treatment, it could be reasonable to shorten the standard period of deepTMS treatment from 4 to 2 weeks, expecting a reduction of dropped-out patients and thus optimizing the treatment outcome.

Keywords: deep transcranial magnetic stimulation, transcranial magnetic stimulation, electroconvulsive therapy, pharmacoresistant unipolar depression

\section{Introduction}

Major depressive disorder (MDD) is a chronic disorder characterized by single and recurrent episodes. As established by converging neuropsychological, biochemical, neuroimaging, and postmortem evidence, depression is unlikely to be a disease of a single brain region or neurotransmitter system. Rather, it is now generally viewed as a system-level disorder affecting integrated pathways. ${ }^{1-5}$ Notwithstanding the therapeutic armament 
available to clinicians, approximately $30 \%$ of patients remain symptomatic despite standard interventions; this group is considered to have treatment-resistant depression., ${ }^{6,7}$

Biological treatments are considered additional options for the treatment of resistant bipolar depression. Controversial data exist about the efficacy and tolerability of three of the most-used somatic treatments: electroconvulsive therapy (ECT), transcranial magnetic stimulation (rTMS), and deep transcranial magnetic stimulation (deepTMS).

ECT is considered an effective alternative for pharmacoresistant patients, even if it necessitates administering general anesthetic, induces a seizure, or may cause memory and learning impairments. ${ }^{8-10}$ rTMS is a noninvasive technique used to apply magnetic pulses to the brain through an electromagnetic coil placed above the patient's scalp, inducing electrical activity in the underlying cortical tissue that can result in localized neuronal depolarization; it has been proposed as a refined alternative. ${ }^{11}$ DeepTMS is currently being evaluated as a treatment option in major depression and has been shown to be a safe and effective procedure. ${ }^{12-16}$ DeepTMS coils are designed to maximize the electrical field deep in the brain by the summation of separate fields projected into the skull from several points around its periphery, while minimizing the accumulation of electrical charge on the surface of the brain. ${ }^{17}$

The aim of this review is to investigate and to compare the efficacy and tolerability of these three techniques in patients with pharmacoresistant unipolar depression. We performed a systematic comparison of the results of those studies where these three techniques were used to treat unipolar drug-free depressed patients. This kind of selection was made to obtain the best evaluation of the therapeutic efficacy of ECT, rTMS, and deepTMS, considering the complete absence of pharmacologic interference.

\section{Methods}

The first step of this study was a selective literature search. Three independent reviewers extracted data and assessed the quality of methodological reporting of selected studies using data extraction forms. Our key search terms were major depression, resistant depression, transcranial magnetic stimulation, rTMS, TMS, deepTMS, deep transcranial magnetic stimulation, ECT, and electroconvulsive therapy. Prospective studies evaluating the effects of the three techniques on mood and cognition in patients with major depression were included. Inclusion criteria for studies were:

1. articles written in English

2. diagnosis of MDD according to the Diagnostical and Statistical Manual of Mental Disorders III, IV, or IV-TR ${ }^{18}$
3. an explicit definition of treatment-resistant depression that included at least one failed trial of an antidepressant drug

4. patients not treated with any kind of psychotropic drug (with the exception of occasional use of benzodiazepines)

5. rTMS given at high frequencies $(>1 \mathrm{~Hz})$ and delivered to left dorsolateral prefrontal cortex (DLPFC)

6. use of deepTMS at high frequencies $(>1 \mathrm{~Hz})$ at any localization

7. use of ECT given at any intensity and localization

8. mood effects assessed by the same continuous mood scale: Hamilton Depression Rating Scale (HDRS) 17 or 24 items

9. studies published within the last 20 years.

The first outcome was the clinical response, defined as a percentage improvement of HDRS evaluated at three different timepoints: pretreatment (T0), after 2 weeks of treatment (T1), and after 4 weeks of treatment (T2). The second outcome was the evaluation of neuropsychological effects of the three different techniques. The third outcome was the evaluation of the number of remitted patients. Tolerability was the fourth outcome. It was evaluated by examining the number of dropped-out patients.

\section{Data analysis}

The sample was divided into three groups: (1) patients treated with ECT (160 patients), (2) patients treated with rTMS (211 patients), and (3) patients treated with deepTMS (58 patients) (Figure 1).

\section{Clinical response}

Clinical response was defined as a decrease on HDRS.

The Fisher-Snedecor F test was used to test the heterogeneity of the data. Using this test, the null hypothesis

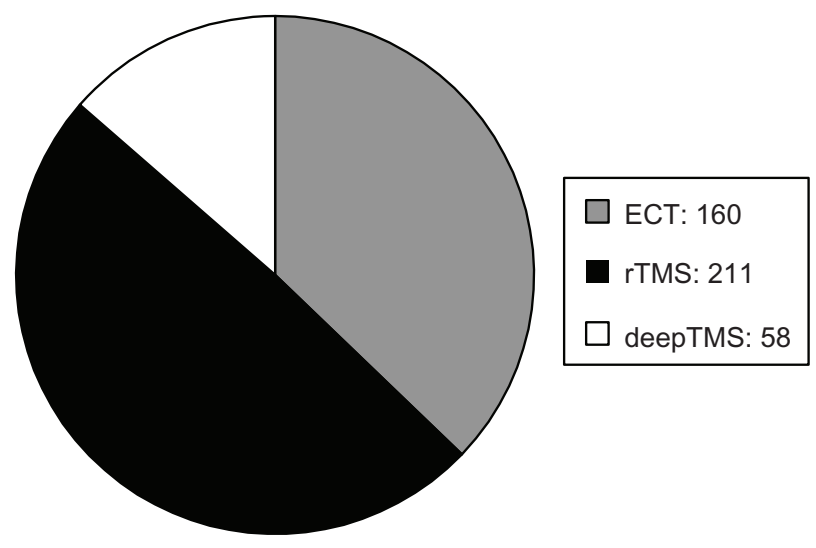

Figure I Number of enrolled patients.

Abbreviations: ECT, electroconvulsion therapy; rTMS, transcranial magnetic stimulation; deepTMS, deep transcranial magnetic stimulation. 
of the equality of the averages was refused $(P \leq 0.05$; confidence interval [CI] 95\%).

We calculated the percentage variation of the HDRS average scores between baseline (T0), 2 weeks of treatment (T1), and 4 weeks of treatment (T2) with each technique. Because of the fact that 307 patients were evaluated with HDRS-24 items and 122 patients were evaluated with HDRS17 items, the group of ECT-treated patients was divided into two subgroups: one of 96 patients where symptoms were evaluated with HDRS-24, and one of 64 patients where symptoms were evaluated with HDRS-17; the group of rTMS-treated patients was also divided into two subgroups: one of 153 patients where symptoms were evaluated with HDRS-24, and one of 58 patients where symptoms were evaluated with HDRS-17.

In all patients treated with deepTMS, depressive symptoms were evaluated with HDRS-24 items.

\section{Neuropsychological effects}

We calculated the percentage variation of average scores of the Mini-Mental State Examination (MMSE), ${ }^{19}$ Weschler Adult Intelligence Scale-Revised (WAIS-R), ${ }^{20}$ Wechsler Memory Scale (WMS), ${ }^{21}$ and Rivermead Behavioral Memory Test (RBMT) ${ }^{22}$ after 2 and 4 weeks of treatment with ECT and rTMS. We also calculated the percentage variation of the Cambridge Neuropsychological Test Automated Battery $(\mathrm{CANTAB})^{23}$ average scores after 2 and 4 weeks of treatment with deepTMS.

\section{Remission rate}

Remission was defined as an absolute HDRS-24 score of $\leq 11$ or as an absolute HDRS-17 score of $\leq 8$.

\section{Tolerability}

The fourth outcome of the study was to evaluate the tolerability of each technique. To do this, we calculated the percentage of dropped-out patients in each group of patients.

\section{Results \\ Included studies}

Only nine studies met our inclusion criteria (Table 1). A total of 160 patients were treated with ECT. ${ }^{24-27}$ Among these, the standard bifrontotemporal (bilateral) placement of electrodes was used in 50 patients, ${ }^{28}$ the d'Elia placement of electrodes was used in $46,{ }^{29}$ and treatment began with right unilateral ECT in 64. If there was no antidepressant response after six ECT treatments, bilateral ECT was performed (Table 1). Two hundred and eleven patients were treated with rTMS..$^{25,26,30-32}$ Of these, 28 received ten sessions of rTMS treatment, 40 received 20 sessions of rTMS treatment, and 143 received 30 sessions of rTMS treatment (Table 1). Fifty-eight patients were treated with deepTMS (Table 1). ${ }^{13,14}$

In the 96 patients treated by Sackeim et al, the seizure threshold was quantified at the time of the first and last treatment with the empirical titration procedure. ${ }^{24}$ Electrical stimulations were administered at subconvulsive levels of increasingly higher intensity until a generalized tonic-clonic seizure of adequate duration was induced. The frequency of brief pulses (range, 20-140 Hz) was the primary electrical variable manipulated, because the device used for electroconvulsive therapy had greater range and sensitivity in the frequency domain than in pulse width, current intensity, or in the duration of the stimulus. The tourniquet method and two channels of prefrontal electroencephalography

Table I Included studies

\begin{tabular}{|c|c|c|c|c|}
\hline Study & Technique & Stimulation & Patients, $\mathbf{n}$ & Duration \\
\hline Sackeim et $\mathrm{al}^{24}$ & ECT & Right unilateral and bilateral; convulsive threshold or 2.5 times threshold & 96 & 4 weeks \\
\hline Rosa et $\mathrm{al}^{25}$ & $\mathrm{ECT}$ & $\begin{array}{l}\text { Right unilateral at a mean charge of } 4.5 \text { times the threshold. If there } \\
\text { was no antidepressant response after } 2 \text { weeks, bilateral ECT was } \\
\text { performed. At I. } 5 \text { times the threshold }\end{array}$ & 15 & 4 weeks \\
\hline Grunhaus et $\mathrm{al}^{26}$ & $\mathrm{ECT}$ & $\begin{array}{l}\text { Unilateral up to } 2.5 \text { times the threshold. If there was no antidepressant } \\
\text { response after } 2 \text { weeks, bilateral ECT was performed }\end{array}$ & 20 & 4 weeks \\
\hline Dannon and Grunhaus ${ }^{27}$ & ECT & $\begin{array}{l}\text { Unilateral up to } 2.5 \text { times the threshold. If there was no antidepressant } \\
\text { response after } 2 \text { weeks, bilateral ECT was performed }\end{array}$ & 29 & 4 weeks \\
\hline O'Reardon et $\mathrm{al}^{30}$ & rTMS & $10 \mathrm{~Hz}$; left DLPFC; I20\% of motor threshold & 143 & 6 weeks \\
\hline Berman et $\mathrm{a}^{31}$ & rTMS & $20 \mathrm{~Hz}$; left DLPFC; $80 \%$ of motor threshold & 10 & 2 weeks \\
\hline Grunhaus et $\mathrm{al}^{26}$ & rTMS & $10 \mathrm{~Hz}$; left DLPFC; $90 \%$ of motor threshold & 20 & 4 weeks \\
\hline Baeken et $\mathrm{al}^{32}$ & rTMS & $10 \mathrm{~Hz}$; left DLPFC; II0\% of motor threshold & 18 & 2 weeks \\
\hline Rosa et $\mathrm{al}^{25}$ & rTMS & $10 \mathrm{~Hz}$; left DLPFC; I00\% of motor threshold & 20 & 4 weeks \\
\hline Levkovitz et $\mathrm{al}^{13}$ & DeepTMS & $20 \mathrm{~Hz}$; left and bilateral DLPFC; II $0 \%$ and $120 \%$ of motor threshold & 53 & 4 weeks \\
\hline Rosenberg et $\mathrm{al}^{14}$ & DeepTMS & $20 \mathrm{~Hz}$; left DLPFC; I20\% of motor threshold & 5 & 4 weeks \\
\hline
\end{tabular}

Abbreviations: ECT, electroconvulsion therapy; rTMS, transcranial magnetic stimulation; DLPFC, dorsolateral prefrontal cortex. 
were used to assess the duration of seizures. In the first five treatments, the criterion for an adequate generalized seizure was at least 25 seconds of tonic-clonic movement or 30 seconds of electroencephalographic seizure activity. After the fifth treatment, the cut-off values were reduced to 20 and 25 seconds, respectively. This change was made to account for the spontaneous decrease in seizure duration that occurs during electroconvulsive therapy. Forty-six of these patients received a low-dose treatment, where the electrical intensity that resulted in a generalized seizure in the first session was the one administered at the next treatment. If this intensity proved adequate, a lower intensity was used for the next treatment. In the low-dose groups, this procedure was followed throughout the treatment course. The other 50 patients received high-dose treatment, where at the second and subsequent sessions each patient received an electrical intensity stimulus 2.5 times the threshold identified in the first treatment, except that at the time of the last treatment the seizure threshold was determined again (Table 1).

In the 29 patients treated by Dannon et al, ${ }^{27}$ seizure threshold was determined for all of them during the first ECT using Sackeim et al's method. ${ }^{33}$ The following ECT treatments were performed at 2.5 times the threshold energy and charge was titrated upward every second or third treatment to maintain a seizure length of 25 seconds. Seizure duration was monitored with both the cuff and electroencephalographic methods (Table 1).

O'Reardon et al performed a double-blind, multicenter study on 301 medication-free patients with major depression who had not benefited from prior treatment. ${ }^{30}$ These patients were randomized to active or sham TMS conditions. Sessions were conducted five times per week with TMS at 10 pulses/second, $120 \%$ of motor threshold (MT), 3000 pulses/session, for 4-6 weeks. The MT estimation was repeated weekly by visual observation of thumb or other finger movement (Table 1).

Rosa et al performed a study on 35 patients aged between 18 and 65 years. ${ }^{25}$ Patients were randomized to receive ECT or rTMS to the left DLPFC according to a computer-generated list. rTMS sessions were performed five times per week for 4 weeks (total of 20 sessions). The following parameters were used: site of stimulation, left DLPFC; intensity, 100\% MT; frequency, $10 \mathrm{~Hz}$; trains of 10 seconds and intertrain interval of 20 seconds; 25 trains per session. Therefore, a total of 2500 pulses were administered each session (overall total of 50,000 pulses). MT was defined as the lowest TMS intensity required to elicit motor-evoked potentials (MEP) of
$0.05 \mathrm{mV}$ in the contralateral resting abduttor pollicis brevis (APB) muscle in at least five of ten trials with the coil over the optimal scalp position. The ECT treatment began with right unilateral ECT. If there was no antidepressant response after 2 weeks, bilateral ECT was performed. In the first treatment, seizure threshold was calculated according to the method of limits. ${ }^{34}$ In the following treatments, a mean charge of 4.5 times the threshold was given three times a week (Monday, Wednesday, and Friday). If a patient was transferred to bilateral ECT, a mean charge of 1.5 times the threshold was given (Table 1).

In the study by Grunhaus et al, 40 patients were included. ${ }^{26}$ Patients were assigned to the rTMS or ECT groups. ECT was performed according to established protocols. The titration of electrical charge was performed in all cases following the method of limits during the first ECT treatment. Additional treatments were performed at 2.5-times threshold charge. Treatments with ECT were continued until the treating physician considered that a therapeutic response had been obtained or that no further therapeutic benefit was to be expected. Patients included in this study were required to have had at least six ECT treatments, unless the course was suspended due to an early therapeutic response. Thirteen of these patients were treated unilaterally, and seven patients were treated bilaterally. Patients switching from unilateral to bilateral treatment received a mean of five additional bilateral treatments. Repetitive TMS was performed over the left DLPFC at 90\% MT. Patients were treated with 20 sessions (five times per week for 4 weeks) of $10 \mathrm{~Hz}$ (1200 pulses per treatment day) at 90\% MT. MT was determined in all individuals following the methods described by Rossini and Rossi. ${ }^{35}$ The tapering process was completed in all cases within 3 days (Table 1).

In the study by Berman et al, 20 depressed subjects were assigned in a randomized double-blind manner to receive either active $(20 \times 2$ second trains of $20 \mathrm{~Hz}$ stimulation with 58 second intervals, delivered at $80 \%$ MT with the figure-of-eight coil positioned over the left DLPFC) or sham rTMS. ${ }^{31}$ MT was determined daily and was defined at the point of maximal stimulation for the right APB or other hand muscles, as visually detected. These sequences were applied during ten consecutive weekdays (Table 1).

In the study conducted by Baeken et al, 19 patients were treated with rTMS. ${ }^{32}$ Before each application, the resting MT of each subject was determined using electromyography. A stimulation intensity of $110 \%$ of the subject's MT of the right APB muscle was used (Table 1). One patient dropped out before the second week of treatment. 
In the studies by Levkovitz et al and Rosenberg et al, each subject was treated with one of three versions of the H-coil (H1, H2, and H1L), similar in external appearance and acoustic properties when actively pulsed. ${ }^{13,14}$ The H1-coil was designed to stimulate deep prefrontal brain regions, preferentially in the left hemisphere. Fifteen patients received the treatment with $\mathrm{H} 1$-coil at $120 \%$ of the measured MT. The H2-coil was designed to stimulate deep prefrontal brain regions bilaterally, with no lateral preference. 18 patients received the treatment with $\mathrm{H} 2$-coil at $120 \%$ of the measured MT. The H1L-coil was designed to stimulate deep prefrontal brain regions unilaterally, exclusively in the left hemisphere. Six patients were treated with H1L-coil at $110 \%$ of the measured MT while ten patients were treated with H1L-coil at $120 \%$ of the measured MT. To localize the optimal placement, suprathreshold intensities were applied around the hand motor area (tested at increments of $1 \mathrm{~cm}$ ) and the spot inducing the greatest motor response was marked. To refine the "hot" spot for APB activation, the intensity was reduced until the MEP was $<100 \mathrm{mV}$ and motor responses were tested around the marked spot searching for placement for inducing the maximal response. The MT was measured by using single pulse mode, applying one pulse every $5-10$ seconds and recording electrical activity in the APB by using surface electrodes. MT was defined before each daily session as the lowest intensity of stimulation able to produce MEP of at least $50 \mathrm{mV}$ in three of six trials. The coil was placed $5.5 \mathrm{~cm}$ anterior to the motor spot (over the prefrontal cortex). The treatment was delivered in trains of $20 \mathrm{~Hz}, 5$ days per week for 4 weeks. Each TMS session consisted of 42, 2-second trains, with an intertrain interval of 20 seconds (ie, a total of 1680 pulses delivered during a 15-minute daily session) (Figure 1).

A total of 96 ECT-treated patients were assessed by HDRS-24 items before the treatment, after six ECT treatments, and at the end of the treatment to evaluate depressive symptoms; 35 ECT-treated patients by HDRS-17 items before the treatment, after six ECT treatments, and at the end of the treatment; 29 ECT-treated patients by HDRS-17 items before and at the end of the treatment.

Among the patients treated with rTMS, 143 were assessed by HDRS-24 items before the treatment, after 4 weeks, and after 6 weeks of treatment to evaluate depressive symptoms; ten patients were assessed by HDRS-24 items before the treatment and after 2 weeks; 19 patients were assessed by HDRS-17 items before the treatment and after 2 weeks. Forty patients received HDRS-17 items before the treatment, after 2 weeks, and after 4 weeks of treatment.
All 58 patients treated with deepTMS were assessed by HRSD-24 items before the treatment, after 2 weeks, and after 4 weeks of treatment to evaluate depressive symptoms.

All 160 patients treated with ECT were assessed by neuropsychological tests focusing on anterograde and retrograde memory. Of these patients, 116 received modified versions of the MMSE before the treatment, after six ECT treatments, and at the end of the course. Of these, 29 received modified versions of the MMSE before and at the end of the treatment and 15 received several tests including subsections of the WAIS-R (vocabulary and cubes), subsections of the WMS (digit span), and the RBMT.

A total of 40 patients treated with rTMS received neuropsychological tests focusing on anterograde and retrograde memory; 20 received MMSE to evaluate cognitive condition before and after the treatment while the other 20 patients received tests including subsections of the WAIS-R (vocabulary and cubes), subsections of the WMS (digit span), and the RBMT.

All 53 patients treated with deepTMS received computerized cognitive assessments using the CANTAB tests at baseline, after 2 weeks, and after 4 weeks of treatment. Each subject was administered the tests in a pseudo-randomized fashion and in a controlled environment. The neuropsychological battery was designed to differentiate dorsolateral, superior medial, and ventrolateral functions potentially affected by deepTMS treatment.

\section{Clinical response}

\section{Clinical response from $\mathrm{TO}$ to $\mathrm{TI}$}

In ECT-treated patients, HDRS-24 decreased by $22.83 \%$ from T0 to T1 (Figure 3), while HRSD-17 decreased by $30.00 \%$. In rTMS-treated patients, HDRS-24 decreased by $33.69 \%$ from T0 to T1, while HRSD-17 decreased by $32.37 \%$. In deepTMS-treated patients, HDRS-24 decreased by $43.81 \%$ from $\mathrm{T} 0$ to $\mathrm{T} 1$ (Figure 3).

These results indicate that after 2 weeks of treatment, deepTMS seems to be more effective than ECT and rTMS in decreasing HDRS-24 score; moreover, ECT seems to be more effective than rTMS.

\section{Clinical response from $\mathrm{TI}$ to $\mathrm{T} 2$}

In ECT-treated patients, HDRS-24 decreased by $21.60 \%$ from T1 to T2 (Figure 3), while HDRS-17 decreased by $18.61 \%$. In rTMS-treated patients, HDRS- 17 decreased by $12.55 \%$ from T1 to T2. In deepTMS-treated patients, HDRS-24 decreased by $0.38 \%$ from $\mathrm{T} 1$ to $\mathrm{T} 2$ (Figure 3 ).

These results suggest that between the second and fourth week of treatment ECT is more effective than rTMS 


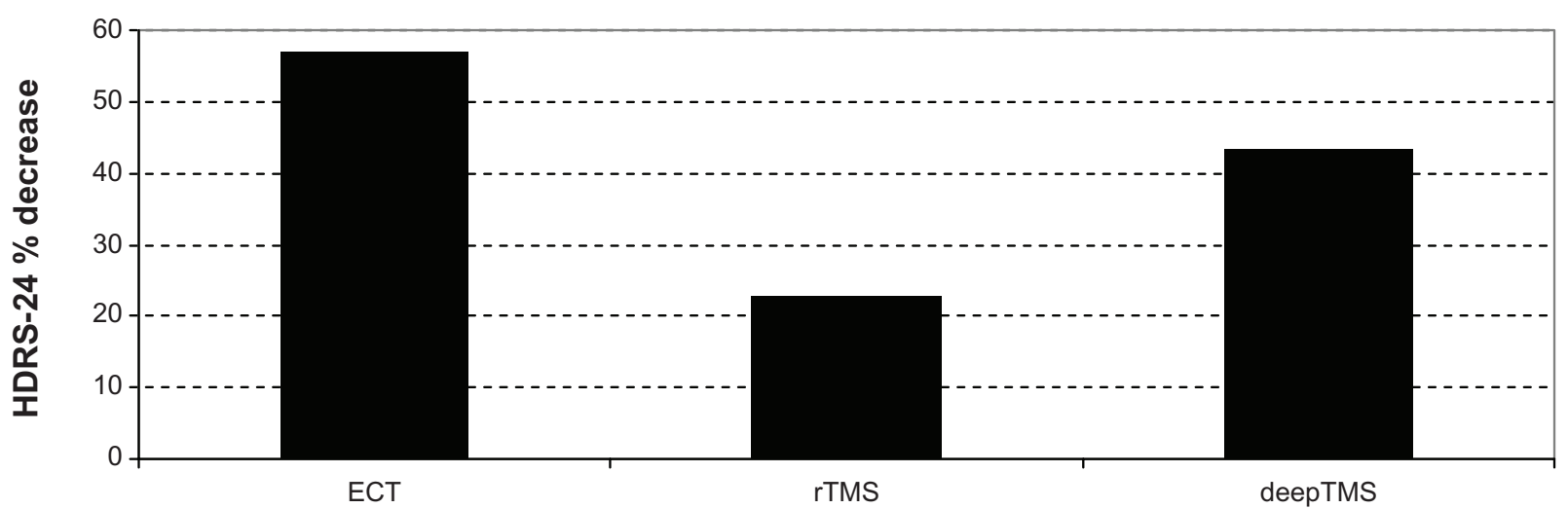

Figure 2 HDRS-24\% decrease in patients treated with ECT, rTMS, and deepTMS from baseline to the fourth week of treatment.

Abbreviations: HDRS, Hamilton Depression Rating Scale; ECT, electroconvulsion therapy; rTMS, transcranial magnetic stimulation; deepTMS, deep transcranial magnetic stimulation.

and deepTMS in decreasing HDRS-24 score; moreover, rTMS is more effective than deepTMS.

\section{Clinical response from T0 to T2}

In ECT-treated patients, HDRS-24 decreased by $56.96 \%$ from T0 to T2 (Figures 2 and 3), while HDRS-17 decreased by $42.84 \%$. In rTMS-treated patients, HDRS-24 decreased by $22.25 \%$ from T0 to T2 (Figure 2), while HDRS- 17 decreased by $42.56 \%$. In deepTMS-treated patients, HDRS24 decreased by $43.59 \%$ from T0 to T2 (Figures 2 and 3).

These results suggest that after 4 weeks of treatment ECT is more effective than deepTMS and rTMS in decreasing HDRS-24 score; moreover, deepTMS is more effective than rTMS (Figure 2).

\section{Neuropsychological effects}

Cognitive condition was the second outcome considered in this study. The cognitive assessment was made using different evaluation methods: MMSE, CANTAB, WAIS-R, WMS, and RBMT.
Neuropsychological effects in ECT-treated patients Fifteen of the ECT-treated patients presented the following percentage variations of average scores (Figures 4 and 5):

- Total vocabulary (WAIS-R): T0-T1: $-7.59 \%$; T1-T2: +4.81\%; T0-T2: $-3.14 \%$.

- Total cubes (WAIS-R): T0-T1:-1.88\%; T1-T2: +9.61\%; T0-T2: +7.54.

- Estimated IQ (WAIS-R):T0-T1:-0.10\%;T1-T2:-0.10\%; T0-T2: $-0.21 \%$.

- Direct Digits (WMS): T0-T1:+10.39\%; T1-T2:-23.52\%; T0-T2: $-15.58 \%$.

- Indirect Digits (WMS): T0-T1:-18.18\%; T1-T2:+4.48\%; T0-T2: $-14.54 \%$.

- Numbers (WAIS-R): T0-T1:-1.51\%; T1-T2: $-13.84 \%$; T0-T2: $-15.15 \%$.

- RBMT profile: T0-T1: $-22.53 \%$; T1-T2: $+13.63 \%$; T0-T2: $-11.97 \%$.

Of the ECT-treated patients, 145 were evaluated by MMSE from T0 to T2. The average score increased by $7.01 \%$ (Figure 5).

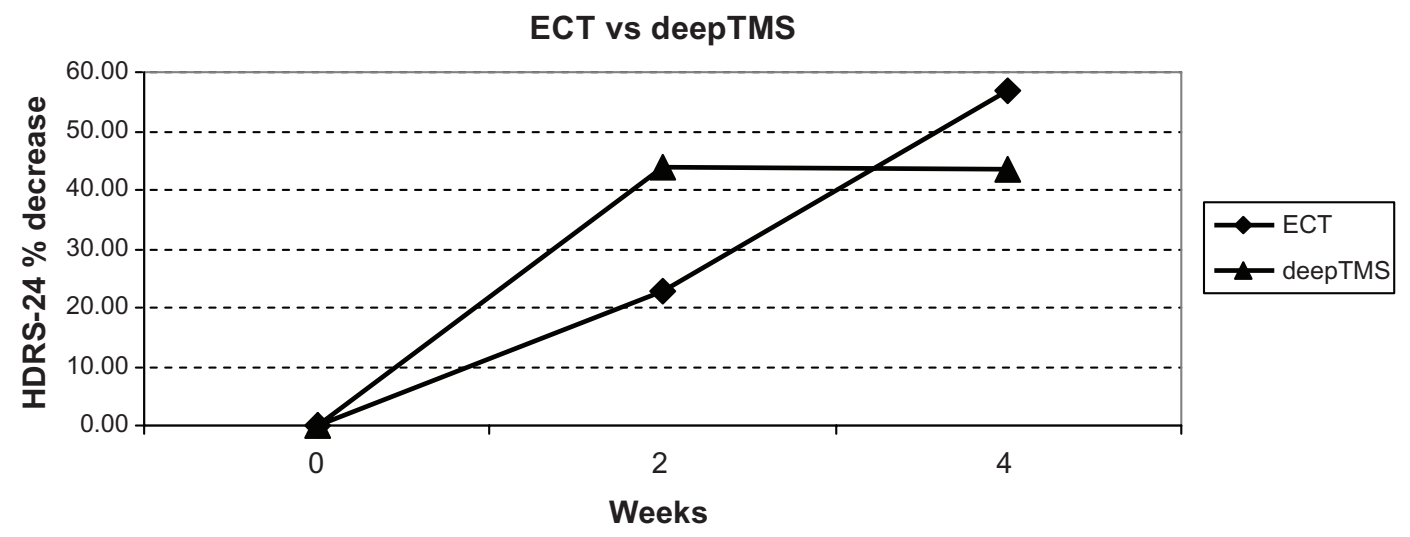

Figure 3 HDRS-24\% decrease in patients treated with ECT and deepTMS after 2 and 4 weeks of treatment.

Abbreviations: HDRS, Hamilton Depression Rating Scale; ECT, electroconvulsion therapy; deepTMS, deep transcranial magnetic stimulation. 


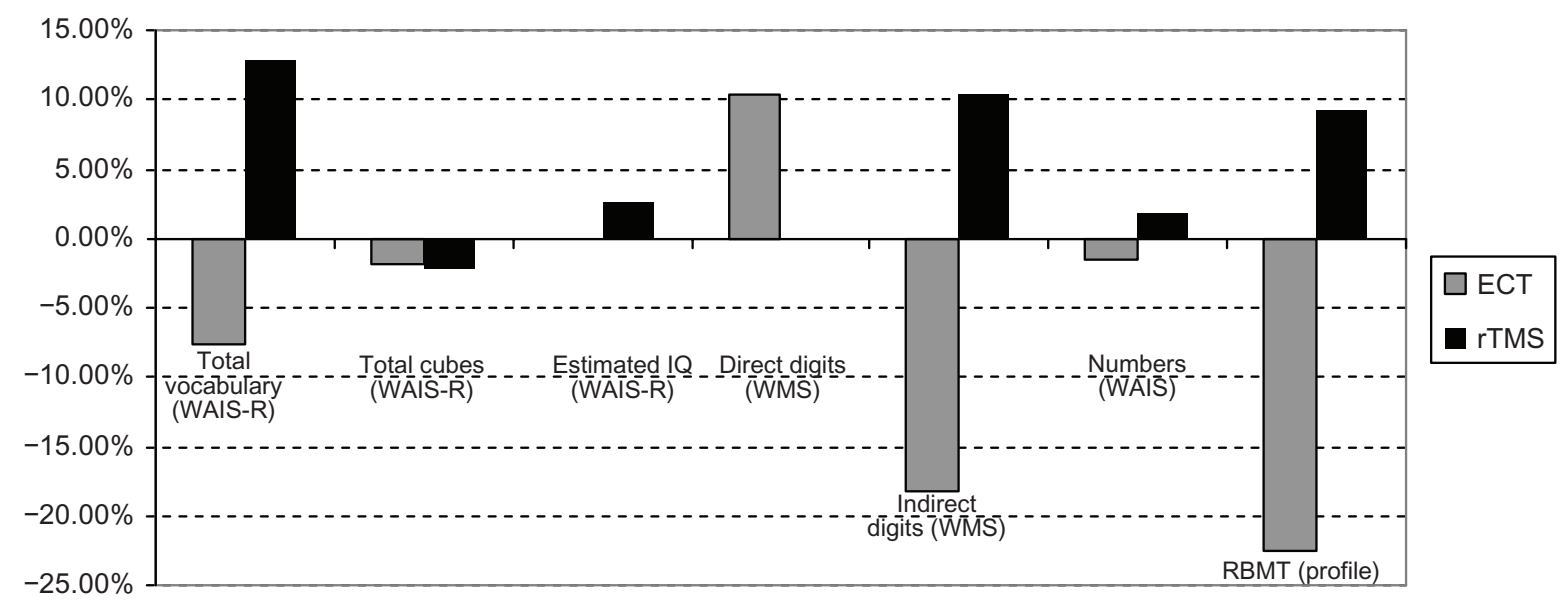

Figure 4 Cognitive variations in patients treated with ECT and rTMS from T0 to TI (2 weeks of treatment).

Abbreviations: ECT, electroconvulsion therapy; RBMT, Rivermead Behavioral Memory Test; rTMS, transcranial magnetic stimulation; WAIS, Weschler Adult Intelligence Scale; WAIS-R, Weschler Adult Intelligence Scale-Revised; WMS, Wechsler Memory Scale.

\section{Neuropsychological effects in rTMS treated patients} Twenty of the rTMS-treated patients were evaluated by MMSE from $\mathrm{T} 0$ to $\mathrm{T} 2$. The average score increased by $0.71 \%$ (Figure 5). The 20 rTMS-treated patients presented the following percentage variations of average scores (Figures 4 and 5):

- Total vocabulary (WAIS-R): T0-T1: +12.80\%; T1-T2: $+0.23 \%$; T0-T2: $+13.06 \%$.

- Total cubes (WAIS-R): T0-T1:-2.21\%; T1-T2:+24.43\%; T0-T2: $+21.68 \%$.

- Estimated IQ (WAIS-R):T0-T1:+2.61\%; T1-T2: +2.12\%; $\mathrm{T} 0-\mathrm{T} 2:+4.71 \%$.

- Direct Digits (WMS): T0-T1: 0.00\%; T1-T2: $-3.17 \%$; T0-T2: $-3.17 \%$.

- Indirect Digits (WMS): T0-T1: +10.41\%; T1-T2: 0.00\%; T0-T2: $+10.41 \%$.
- Numbers (WAIS-R): T0-T1: +1.75\%; T1-T2: $+0.86 \%$; $\mathrm{T} 0-\mathrm{T} 2:+2.63 \%$.

- RBMT profile: T0-T1: +9.14\%; T1-T2: $-3.35 \%$; T0-T2: $+5.48 \%$.

\section{Neuropsychological effects in deepTMS} treated patients

A total of 53 patients treated with deepTMS received CAN$\mathrm{TAB}$ neuropsychological assessment at T0 and T2. They presented the following percentage variations of average scores:

- Sustained attention: $+2.29 \%$.

- Visuospatial memory: $+15.72 \%$.

- Cognitive planning: $+6.17 \%$.

- Spatial memory: $+15.89 \%$.

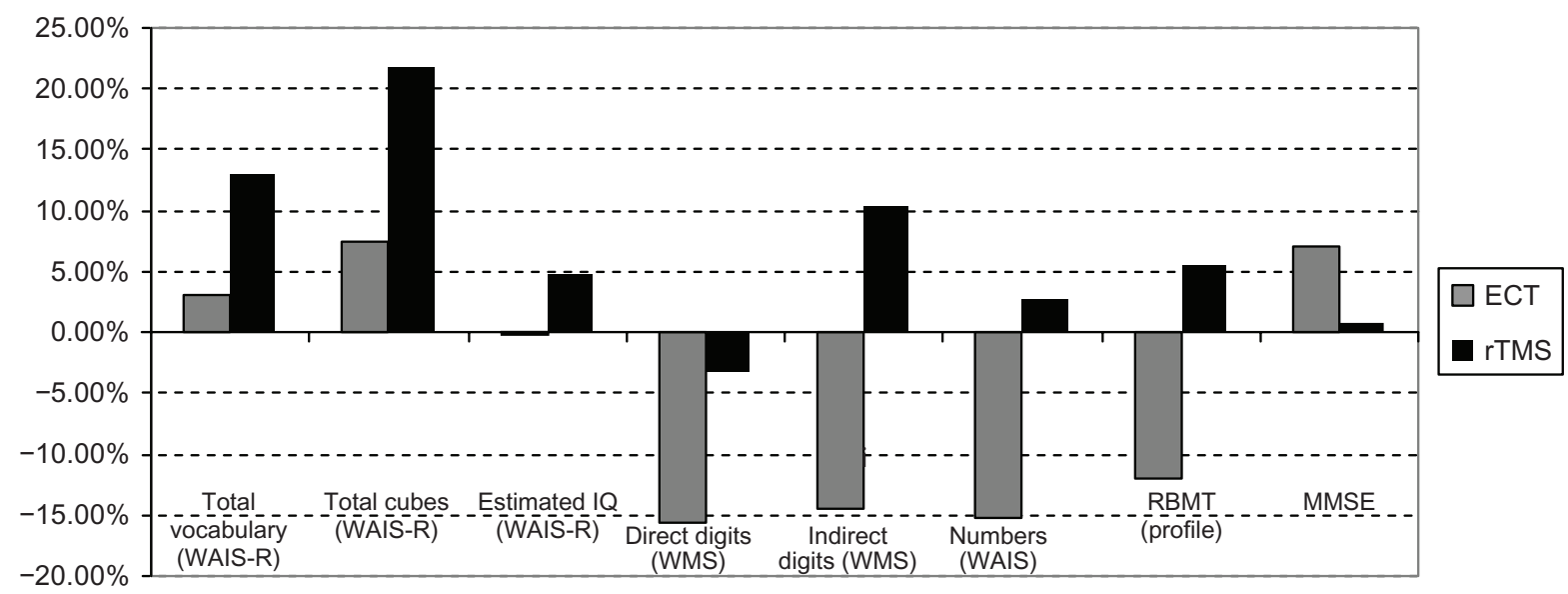

Figure 5 Cognitive variations in patients treated with ECT and rTMS from T0 to T2 (4 weeks of treatment).

Abbreviations: ECT, electroconvulsion therapy; RBMT, Rivermead Behavioral Memory Test; rTMS, transcranial magnetic stimulation; WAIS, Weschler Adult Intelligence Scale; WAIS-R, Weschler Adult Intelligence Scale-Revised; WMS, Wechsler Memory Scale. 


\section{Remission rate}

Remission rate was the third outcome of the study. Remission was defined as an HDRS-24 score of $\leq 11$ or as an HDRS17 score of $\leq 8$. Remission was achieved by $28.57 \%$ of patients treated with ECT, $14.21 \%$ of patients treated with rTMS, and $29.31 \%$ of patients treated with deepTMS (Figure 6). These results suggest that deepTMS and ECT have a comparable efficacy in achieving remission and that both deepTMS and ECT seem to be more effective than rTMS in achieving remission after 4 weeks of treatment.

\section{Tolerability}

Tolerability, measured by the number of dropped-out patients, was the fourth outcome that we highlighted in this study. The following drop-out rates are shown in Figure 7: 9/169 patients dropped out from ECT trials (13.04\%), $16 / 227$ patients dropped out from rTMS trials (7.04\%), and 14/58 patients dropped out from deepTMS trials (19.44\%).

These results suggest that rTMS is more tolerable than ECT and deepTMS; moreover, that ECT is more tolerable than deepTMS.

\section{Discussion}

The aim of this review was to compare the efficacy and the tolerability of ECT, rTMS, and deepTMS in pharmacoresistant depressed drug-free patients.

The comparative evaluation of the percentage variations of HDRS average scores from T0 to T2 indicates ECT as the best therapy, followed by deepTMS and rTMS (Figures 2 and 3). The percentage of remitted patients obtained with ECT treatment is the same obtained in the deepTMS group. Both techniques showed a percentage of remitted patients two times higher than rTMS (Figure 6). Even if the percentage of remitted patients was very similar, deepTMS showed a tolerability, measured by the number of dropped-out patients, worse than ECT (Figure 7). Finally, deepTMS showed all its therapeutic potential within the first two weeks of treatment, with almost no further activity from the second to the fourth week of treatment (Figure 3).

Considering the small therapeutic efficacy of deepTMS in the last 2 weeks of treatment, it could be reasonable to shorten the standard period of deepTMS treatment from 4 to 2 weeks, expecting a reduction of dropped-out patients and thus optimizing the treatment outcome.

To our knowledge, data about the relapse risk related to the duration of deepTMS treatment are missing. Such data are needed to establish the best duration of therapy. In fact, data we presented suggest that deepTMS is absolutely the most effective therapy after 2 weeks of treatment.

Regarding cognitive performances, our results stimulate some considerations. The cognitive performances of ECTtreated patients decreased in the first 2 weeks (Figure 4), and slightly increased at the end of treatment (Figure 5). This was different from what happened in patients on rTMS and deepTMS therapy, whose cognitive performances had a stable improvement. Among the three techniques, deepTMS seems to give the best benefits in terms of cognitive performance improvements.

In conclusion, our investigation confirms the great therapeutic power of ECT. Nevertheless, this technique has different drawbacks: it needs hospitalization (which may be very expensive), it necessitates administering general anesthetic, induces a seizure, and may cause cognitive impairments. rTMS provides better tolerability for the

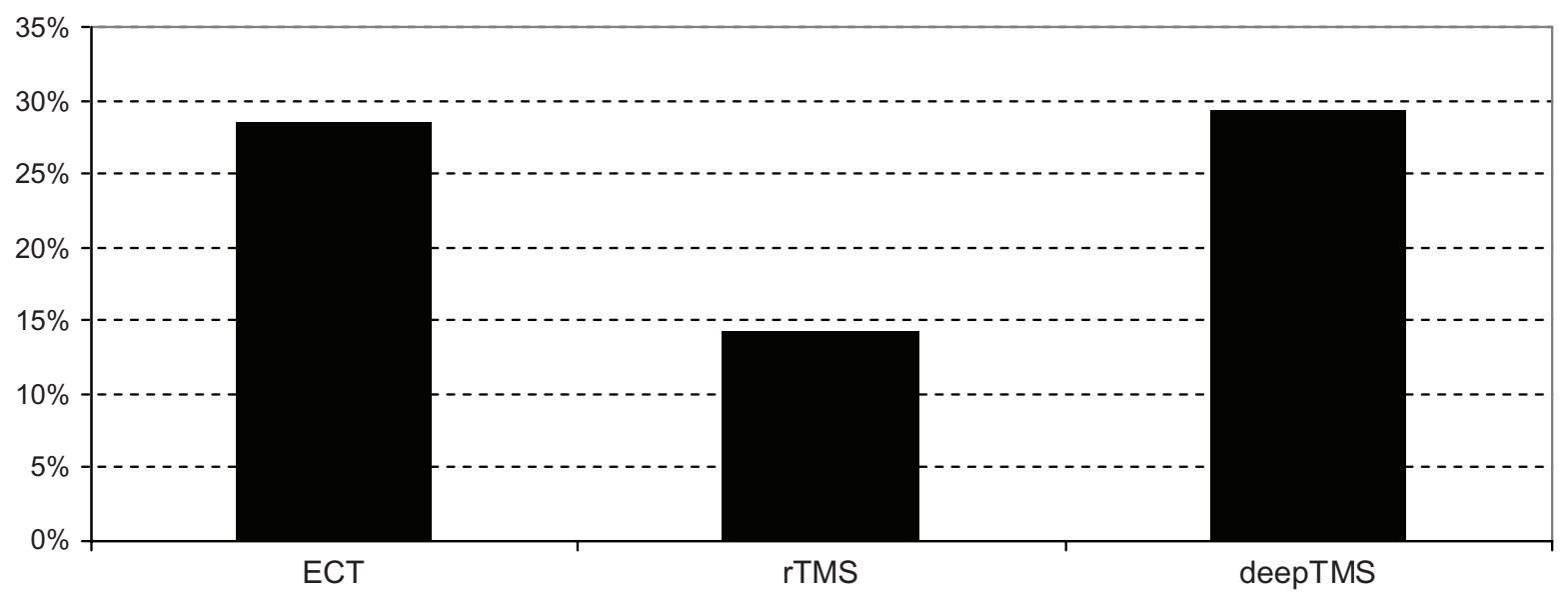

Figure 6 Percentage of remitted patients.

Abbreviations: ECT, electroconvulsion therapy; rTMS, transcranial magnetic stimulation; deepTMS, deep transcranial magnetic stimulation. 


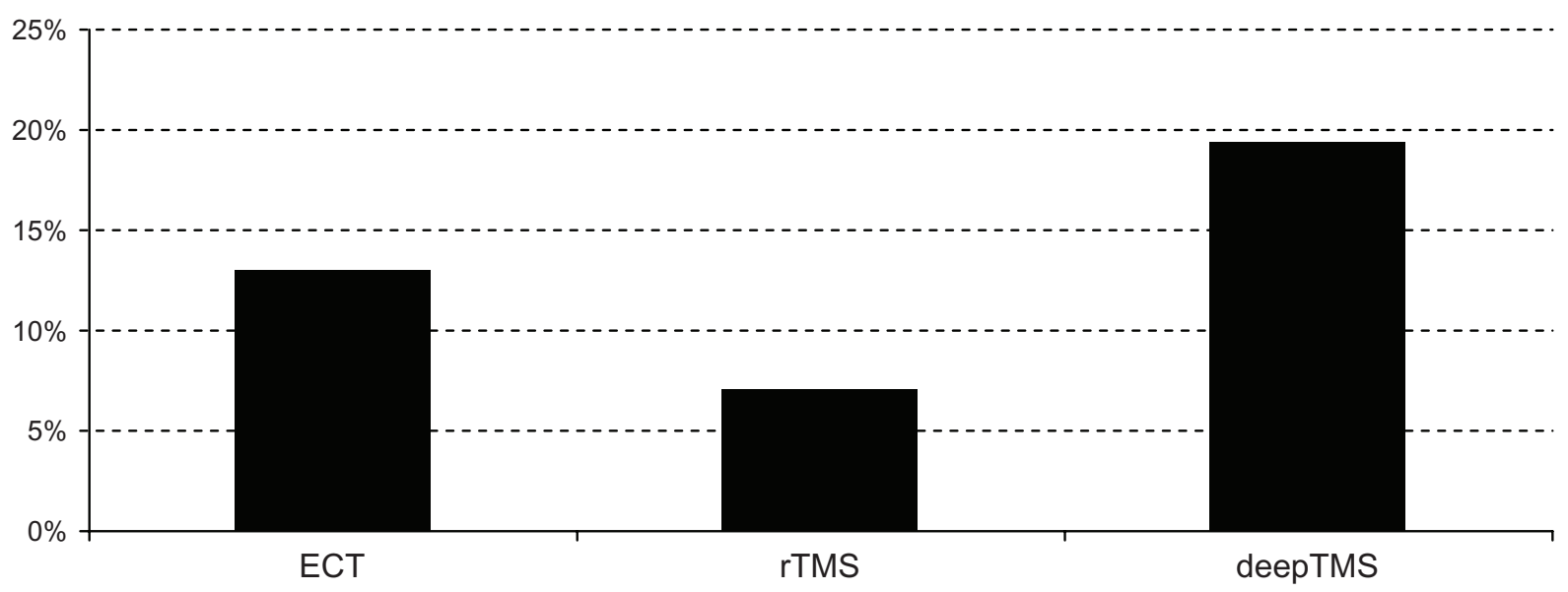

Figure 7 Percentage of dropped-out patients.

Abbreviations: ECT, electroconvulsion therapy; rTMS, transcranial magnetic stimulation; deepTMS, deep transcranial magnetic stimulation.

patients but its therapeutic efficacy is lower. DeepTMS seems to be the only therapy (among the three therapies we analyzed in this review) that provides a substantial improvement in both depressive symptoms and cognitive performances; nevertheless, it is characterized by poor tolerability (greatest number of drop-out patients), which could potentially be avoided by reducing the duration of the treatment.

Our investigation has some limitations. The first of these is the use of HDRS as the only method of evaluating the clinical response of depression. HDRS contains a number of items focused on anxiety and somatic symptoms that do not allow capture of the most specific depressive symptoms. Lack of data regarding the long-term effects of rTMS and deepTMS limits the completeness of our investigation. Some relevant trials may have been unintentionally missed during the literature search. The rigorous inclusion criteria may represent both a weakness and a strength of our study. In fact, the difficulty in collecting a large number of patients and the disproportionate size of the three groups of patients represent an important limitation. On the other hand, rigorous selection provides a better evaluation of the real efficacy of each technique, considering the complete absence of pharmacologic interference. Finally, we are conscious that therapeutic responses in double-blind studies are markedly lower than in open-label studies; the absence of double-blind studies using ECT or deepTMS in drugfree unipolar depressed patients limits the possibility of achieving a definitive conclusion.

\section{Disclosure}

The authors report no conflicts of interest in this work.

\section{References}

1. Manji HK, Drevets WC, Charney DS. The cellular neurobiology of depression. Nat Med. 2001;7:541-547.

2. Mayberg HS. Limbic-cortical dysregulation: a proposed model of depression. J Neuropsychiatry Clin Neurosci. 1997;9:471-481.

3. Nemeroff CB. Recent advances in the neurobiology of depression. Psychopharmacol Bull. 2002;36(Suppl 2):6-23.

4. Nestler EJ, Barrot M, DiLeone RJ, Eisch AJ, Gold SJ, Monteggia LM. Neurobiology of depression. Neuron. 2002;34:13-25.

5. Vaidya VA, Duman RS. Depresssion - emerging insights from neurobiology. Br Med Bull. 2001;57:61-79.

6. Fava M. Diagnosis and definition of treatment-resistant depression. Biol Psychiatry. 2003;53:649-659.

7. Berlim MT, Turecki G. What is the meaning of treatment resistant/ refractory major depression (TRD)? A systematic review of current randomized trials. Eur Neuropsychopharmacol. 2007;17:696-707.

8. Rami-Gonzalez L, Bernado M, Boget T, Salamero M, Gil-Verona JA, Junque C. Subtypes of memory dysfunction associated with ECT: characteristics and neurobiological bases. J ECT. 2001;17:129-135.

9. Lukoyanov NV, Sa MJ, Madeira MD, Paula-Barbosa MM. Selective loss of hilar neurons and impairment of initial learning in rats after repeated administration of electroconvulsive shock seizures. Exp Brain Res. 2004;154:192-200.

10. Zambello F, Vaona A. ECT. A critical review of meta-analyses. Riv Psichiatr. 2009;44(5):337-340.

11. George MS, Lisanby SH, Sackeim HA. Transcranial magnetic stimulation: applications in neuropsychiatry. Arch Gen Psychiatry. 1999;56:300-311.

12. Levkovitz Y, Roth Y, Harel EV, Braw Y, Sheer A, Zangen A. A randomized controlled feasibility and safety study of deep transcranial magnetic stimulation. Clin Neurophysiol. 2007;118:2730-2744.

13. Levkovitz Y, Harel EV, Roth Y, et al. Deep transcranial magnetic stimulation over the prefrontal cortex: evaluation of antidepressant and cognitive effects in depressive patients. Brain Stimul. 2009;2:188-200.

14. Rosenberg O, Shoenfeld N, Zangen A, Kotler M, Dannon PN. DeepTMS in a resistant major depressive disorder: a brief report. Depress Anxiety. 2010;27:465-469.

15. Rosenberg O, Zangen A, Stryjer R, Kotler M, Dannon PN. Response to deepTMS in depressive patients with previous electroconvulsive treatment. Brain Stimul. 2010;3:211-217.

16. Rosenberg O, Isserles M, Levkovitz Y, Kotler M, Zangen A, Dannon PN. Effectiveness of a second deepTMS in depression: A brief report. Prog Neuropsychopharmacol Biol Psychiatry. 2011;35:1041-1044. 
17. RothY, Amir A, LevkovitzY, Zangen A. Three-dimensional distribution of the electric field induced in the brain by transcranial magnetic stimulation using figure-8 and deep H-coils. J Clin Neurophysiol. 2007;24:31-38.

18. American Psychiatric Association. Diagnostic and Statistical Manual of Mental Disorders. Text revision (DSM-IV-TR). 4th ed. Washington DC: American Psychiatric Association; 2000.

19. Folstein MF, Robins LN, Helzer JE. The Mini-Mental State Examination. Arch Gen Psychiatry. 1983;40:812.

20. Wechsler D. Manual for the Wechsler Adult Intelligence Scale-Revised. New York, NY: The Psychological Corporation; 1981.

21. Wechsler D. Wechsler Memory Scale - Revised Manual. San Antonio, TX: The Psychological Corporation; 1987.

22. Wilson BA, Baddeley AD, Cockburn JM. The Rivermead Behavioural Memory Test. 2nd ed. Suffolk: Thames Valley Company; 1991.

23. Falconer DW, Cleland J, Fielding S, Reid IC. Using the Cambridge Neuropsychological Test Automated Battery (CANTAB) to assess the cognitive impact of electroconvulsive therapy on visual and visuospatial memory. Psychol Med. 2010;40:1017-1025.

24. Sackeim HA, Prudic J, Devanand DP, et al. Effects of stimulus intensity and electrode placement on the efficacy and cognitive effects of electroconvulsive therapy. $N$ Engl J Med. 1993;328:839-846.

25. Rosa MA, Gattaz WF, Pascual-Leone A, et al. Comparison of repetitive transcranial magnetic stimulation and electroconvulsive therapy in unipolar non-psychotic refractory depression: a randomized, single-blind study. Int J Neuropsychopharmacol. 2006;9:667-676.

26. Grunhaus L, Schreiber S, Dolberg OT, Polak D, Dannon PN. A randomized controlled comparison of electroconvulsive therapy and repetitive transcranial magnetic stimulation in severe and resistant nonpsychotic major depression. Biol Psychiatry. 2003;53:324-331.
27. Dannon PN, Grunhaus L. Effect of electroconvulsive therapy in repetitive transcranial magnetic stimulation non-responder MDD patients: a preliminary study. Int J Neuropsychopharmacol. 2001;4:265-268.

28. Merkl A, Heuser I, Bajbouj M. Antidepressant electroconvulsive therapy: mechanism of action, recent advances and limitations. Exp Neurol. 2009;219:20-26.

29. d'Elia G. Unilateral electroconvulsive therapy. Acta Psychiatrica Scandinavica. 1970;215(Suppl):1-98.

30. O'Reardon JP, Solvason HB, Janicak PG, et al. Efficacy and safety of transcranial magnetic stimulation in the acute treatment of major depression: a multisite randomized controlled trial. Biol Psychiatry. 2007;62:1208-1216.

31. Berman RM, Narasimhan M, Sanacora G, et al. A randomized clinical trial of repetitive transcranial magnetic stimulation in the treatment of major depression. Biol Psychiatry. 2000;47:332-337.

32. Baeken C, De Raedt R, Santermans L, et al. HF-rTMS treatment decreases psychomotor retardation in medication-resistant melancholic depression. Prog Neuropsychopharmacol Biol Psychiatry. 2010;34: 684-687.

33. Sackeim HA, Decina P, Portnoy PJ, Neeley P, Malitz S. Studies of dosage, seizure threshold and seizure duration in ECT. Biological Psychiatry. 1987;22:249-268.

34. Beyer JL, Weiner RD, Glenn MD. Electroconvulsive Therapy. A Programmed Text. 2nd ed. Washington, DC: American Psychiatric Press; 1998.

35. Rossini PM, Rossi S. Clinical applications of motor evoked potential. Electroencephalogr Clin Neurophysiol. 1998;106:180-194.
Neuropsychiatric Disease and Treatment

\section{Publish your work in this journal}

Neuropsychiatric Disease and Treatment is an international, peerreviewed journal of clinical therapeutics and pharmacology focusing on concise rapid reporting of clinical or pre-clinical studies on a range of neuropsychiatric and neurological disorders. This journal is indexed on PubMed Central, the 'PsycINFO' database and CAS.

\section{Dovepress}

The manuscript management system is completely online and includes a very quick and fair peer-review system, which is all easy to use. Visit http://www.dovepress.com/testimonials.php to read real quotes from published authors. 